\title{
Desarrollo de la competencia técnológica en el marco STEAM para la docencia universitaria: experiencia en el Museu de Belles Arts de València
}

\author{
María Ángeles Carabal Montaguda, Virginia Santamarina Camposb Maria del Val Segarra Oñac y \\ María Blanca de Miguel Molinad \\ aUniversitat Politècnica de València, macamon@crbc.upv.es, bUniversitat Politècnica de València, \\ virsanca@crbc.upv.es, cUniversitat Politècnica de València, maseo@omp.upv.es, y dUniversitat Politècnica de \\ València, bdemigu@omp.upv.es.
}

\begin{abstract}
STEAM is an innovative teaching-learning process based on Science, Technology, Engineering, Arts and Maths competences. Accordingly with this new approach, a team of Fine Arts and Management professors are currently leading an innovative project based on "Applying STEAM Strategies in the Social Sciences and Arts Areas". The objective is to establish links between the different STEAM competences -science, technology, engineering, art and mathematics- as a common element in classrooms, enhancing technological training. This new approach motivates students and connects teaching to present society's needs. In this paper we present an analysis of a teaching experience outside the classroom, developed at the Museu de Belles Arts in Valencia (Spain). In this activity, several tools that use technology with which the students are familiar are used, in which is called "BYOD" (Bring Your Own Device). In this case we analyze the use of the photography as a teaching tool. Some of the characteristics of the activity include free time and different spaces at the museum, DIY -Do it Yourself-, cooperative teamwork, peer learning, integration of theory into practice, flexible thinking and analytical skills. The activity looks to motivate them through gamification. After data collection, debriefing is used for integrating results. We will analyze the times spent in the museum and in the processing of the proposed practice, in addition to the convenience of this type of practice, according to the experience of the students.
\end{abstract}

Keywords: STEAM competences, art, museum, technology, photography, outside classroom activity, Technical University of Valencia

\section{Resumen}

Dentro del proyecto de innovación y mejora educativa "Aplicando estrategias STEAM - Science, Technology, Engineering, Arts and Maths- en las áreas de Ciencias Sociales y Arte”, proponemos en nuestras asignaturas un enfoque integrador de distintos objetivos de aprendizaje. En ellos establecemos vínculos entre las diversas competencias STEAM -ciencia, tecnología, ingeniería, arte y matemáticas- como elemento común en las aulas, potenciando la formación tecnológica, que resulta motivadora para el alumnado y acerca la docencia a la sociedad actual. Participamos en este proyecto profesores de dos departamentos de la Universitat Politècnica de València: Conservación y Restauración de Bienes Culturales y Organización de Empresas, generando sinergias que implementen las competencias STEAM.

El presente artículo realiza un análisis de un experiencia docente fuera del aula, en el Museu de Belles Arts de València. En ella se han empleado herramientas que incluyen el uso de la tecnología con la que están familiarizados, a través de BYOD -Bring Your Own Device-, el uso de la fotografía 
como herramienta docente, la libertad de tiempos y espacios en el mismo museo, a través de DIY Do it Yourself-, el trabajo cooperativo en equipo, el aprendizaje por pares, la integración de la teoría en la práctica, el pensamiento flexible y la capacidad de análisis con una práctica que trata de motivar, con el empleo de la gamificación. Tras la toma de datos, se emplea el debate como herramienta metodológica integradora de resultados. Analizaremos los tiempos empleados en el museo y en el procesado de la práctica propuesta, además de la conveniencia de esta tipología de prácticas, según la experiencia del alumnado.

Palabras clave: competencias STEAM, arte, museo, tecnología, fotografía, actividad fuera del aula, Universitat Politècnica de València

\section{Introducción}

El presente texto va a realizar un análisis de una experiencia innovadora en la que se integran las nuevas tecnologías para su uso docente, mediante el empleo de STEAM (en Ciencia, Tecnología, Ingeniería, Arte y Matemáticas), para un aprendizaje profundo y empírico. La experiencia se centra en la realización de una práctica, encaminada a la profesionalización del alumnado, en la que concurren simultáneamente múltiples herramientas docentes, en la que prima la gamificación, para activar la motivación del alumnado. La práctica se efectuó en el Museu de Belles Arts San Pius V. El alumnado acudió al museo y la práctica se realizó en el horario habitual de la asignatura. Posteriormente, la información obtenida en la pinacoteca fue procesada de manera individual y grupal, para realizar una posterior exposición y debate en el aula, además de un informe final, en el que se activaba la investigación, para comprender el contexto de las diversas resoluciones.

La experiencia consisitió en la identificación de técnicas de dorado en los marcos y fragmentos de retablo, que forman parte del citado espacio expositivo. Asimismo sirvió para ahondar en las metodologías decorativas relacionadas con la materia de estudio, que se asentaba sobre prácticas ya efectuadas en el aula, dándole una perspectiva de realidad a lo estudiado en probetas. La finalidad de la experiencia encierra conceptos complejos, que son muy difíciles de completar en un aula, sin acceso a obras reales. El escenario escogido fue la segunda pinacoteca de España, tras el Museo del Prado, referente museístico en la Comunitat Valenciana, por la diversidad en la casuística de las obras. "En sus fondos destacan obras del gótico internacional, del renacimiento valenciano influenciado por el espíritu Quattrocentista, del barroco español, (...) de la escuela valenciana de los siglos XIX y XX: Sorolla, Benlliure, Pinazo o Degrain.” (Ars Magna, 2020).

El marco de aplicación ha sido la asignatura Introducción a la Conservación y Restauración de Dorados y Policromías (cód.14068) del Grado en Conservación y Restauración de Bienes Culturales de la UPV. La presente actividad se planteó y se desarrolló en enero de 2020. En la asignatura, optativa de cuarto curso había 45 alumnas y alumnos matriculados. El presente texto se presenta en el marco del Equipo de Innovación y Calidad Educativa EICE “AFA-Realidad El proceso de Enseñanza y Aprendizaje Fuera de las Aulas - Baños de Realidad”, del Instituto de Ciencias de la Educación de la UPV.

Las herramientas metodológicas empleadas han sido, entre otras, BYOD -Bring Your Own Device- y DIY -Do It Yourself-. BYOD -Trae Tu Propio Dispositivo- invita a que el alumnado haga uso de sus propios terminales para mantenerse conectados, acceder a datos o completar tareas para sus organizaciones, a través de sus tabletas / lectores electrónicos, teléfonos inteligentes, entre otros (Afreen, 2014). DIY -Hazlo Tú Mismo- consiste en que el alumnado explore la metodología que le resulte más adecuada, dándoles la posibilidad de realizar la práctica -o partes de la práctica- de manera libre. Es una filosofía que nace en el contexto laboral, en la búsqueda de la activación de psicología positiva, orientada al individuo sobre las 
emociones, la autoeficacia y el compromiso (Ouweneel et al., 2013) y se ha extrapolado a la docencia, en experiencias que han demostrado su eficacia, comprendendiendo el contexto del alumnado y reflexionando en la experiencia de aprender con sentido, entre otros (García et al., 2018).

La presente experiencia, asimismo, fomenta el trabajo cooperativo, el aprendizaje por pares, además de la exposición y debate. Con ello se han trabajado, mediante un desarrollo lúdico, contenidos planteados en la asignatura, de relativa complejidad y directamente relacionados con el ámbito laboral, en el último curso de grado. Asimismo se ha dirigido hacia la adquisición de distintas competencias transversales, trabajando su relación con los objetivos de desarrollo sostenible (ODS) (United Nations, 2014).

\section{Objetivos}

\subsection{Objetivo general}

Analizar los resultados de la eficacia de la aplicación de estrategias STEAM, cuyo ámbito de desarrollo son espacios fuera del aula, que pueden convertise en espacios docentes, mediante el uso de la tecnología.

\subsection{Objetivos específicos}

- $\quad$ Evidenciar los resultados obtenidos con medios tecnológicos (competencia Technology), valorando un abanico de posibilidades, mediante la toma de decisiones (competencia Engineering), siendo capaces de aplicar resultados medibles (competencia Science), empleando para ello el análisis artístico (competencia Arts\& Design).

- $\quad$ Analizar la aplicación de ODS en el marco de aplicación de la estrategia docente expuesta.

- Contribuir a generar en el alumnado una visión analítica y real de los contenidos trabajados, para que puedan desarrollar su pensamiento práctico y crítico en posteriores ámbitos laborales, promoviendo la comunicación eficiente.

Promover el trabajo en equipo, el desarrollo de metodologías cooperativas, liderazgo y la resolución de problemas, potenciando la igualdad de condiciones y la paridad.

Transferir los resultados de la experiencia docente a la comunidad universitaria.

\section{Desarrollo de la innovación}

En el desarrollo de la actividad se han empleado las herramientas metodológicas que, combinadas, han generado una morfología completa, con todas las variantes y singularidades que se trataban de trabajar en la propuesta.

Se ha recurrido a metodologías como BYOD -Bring Your Own Device-, DIY -Do It Yourself-, el trabajo cooperativo, el aprendizaje por pares, además de la exposición y debate. El desarrollo lúdico de la actividad ha sido el leitmotiv que ha asegurado su implicación, para aunar contenidos planteados en la asignatura, complejos, desarrollados en los temas 2 -Caracterización, estudio técnico e identificación de metodologías de dorado, plateado y policromía sobre diferentes soportes- y 3 -Principales factores de deterioro- de la asignatura cuatrimestral (Universitat Politècnica de València, 2019).

La herramienta BYOD -Bring Your Own Device- en su variante tecnológica, ha sido diferente del habitual uso en las aulas -que se emplea en mayor medida como acceso a la información- (Afreen, 2014). En este caso, la citada herramienta metodológica se ha empleado como soporte de la propia práctica y para el registro de las actividades propuestas, que serán tratadas más tarde en el aula, a modo de debate y para una posterior encuesta de resultados. Sin esta modalidad sería inviable la realización de la práctica, dado que trabajan directamente sobre el dispositivo.

(cc) EY-NC-ND 2020, Universitat Politècnica de València

Congreso In-Red (2020) 
La metodología DIY -Do It Yourself- es un elemento motivador, que empodera al usuario porque tiene un mayor control (Hurst et al, 2011). En este caso, siguiendo la normativa del museo, se dispusieron grupos de trabajo de cuatro a cinco personas, con total libertad de movimiento, lo que les planteaba la posibilidad de gestionar su propio tiempo, acordándolo con el equipo.

\subsection{Planificación de la actividad}

La estrategia para el desarrollo de la actividad (FIGURA 1) ha sido, el análisis y selección de los ODS a trabajar, la toma de contacto con el museo para analizar las posibilidades organizativas de la práctica, seguido de una sesión de reconocimiento de las técnicas exisentes, valorando su idoneidad con los objetivos de la práctica y la elaboración del material a trabajar, que será facilitado por los alumnos.

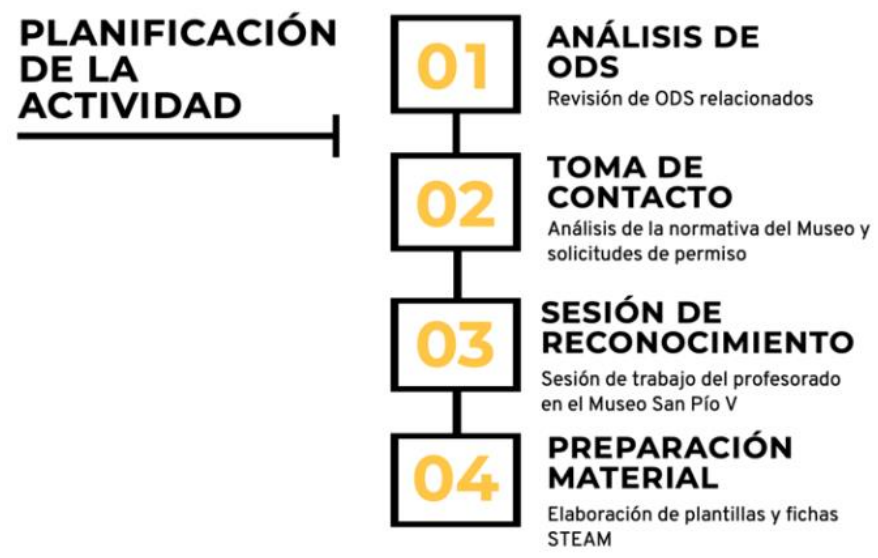

Figura 1. Esquema de la metodología llevada a cabo. Fase 1: planificación. Fuente: elaboración propia.

\subsubsection{Análisis de los ODS relacionados.}

En el proyecto "Aplicando estrategias STEAM en las áreas de Ciencias Sociales y Arte" en el que se enmarca la presente estrategia, dentro de la metodología trabajada, hemos incluido los ODS de cada competencia. En el apartado tecnológico se han seleccionado (United Nations, 2019):

- 4. Educación de calidad. Aumentar considerablemente el número de jóvenes y adultos que tienen las competencias necesarias, en particular técnicas y profesionales, para acceder al empleo, el trabajo decente y el emprendimiento.

- 8. Trabajo decente y crecimiento económico. Lograr niveles más elevados de productividad económica mediante la diversificación, la modernización tecnológica y la innovación, entre otras cosas centrándose en los sectores con gran valor añadido y un uso intensivo de la mano de obra.

- 9. Industria, innovación e infraestructura. Apoyar el desarrollo de tecnologías, la investigación y la innovación nacionales, incluso garantizando un entorno normativo propicio a la diversificación industrial.

- 11. Ciudades y comunidades sostenibles. Redoblar los esfuerzos para proteger y salvaguardar el patrimonio cultural y natural del mundo. 
- 12. Producción y consumo responsable. Alentar al alumnado a que adopte prácticas sostenibles e incorpore información sobre la sostenibilidad en su ciclo de presentación de informes.

Estos mismos objetivos se plantean en la actividad, dada su naturaleza tecnológica e innovadora. Asimismo se prima la ausencia de residuos de la práctica, como papeles borrador, facilitando un formato sostenible. Se ha tratado de aportar la visión del trabajo de otras instituciones, adentrándonos en alguna de las posteriores dedicaciones laborales del alumnado implicado. De igual modo, durante el tiempo de procesado recurrieron a la investigación, en tiempo no presencial, para completar los ítems seleccionados. Todo ello encaminado a la preservación del patrimonio cultural, en concreto de alguno de los ejemplos del patrimonio más valioso para el inventario de la Comunitat Valenciana.

\subsubsection{Toma de contacto con el Museu de Belles Arts.}

El Museu de Belles Arts de València fue muy receptivo con la posibilidad de efectuar la práctica, desde el primer momento, concediendo la realización de la actividad propuesta, siempre y cuando no alterase el correcto funcionamiento de la institución. La normativa del museo incluye una serie de indicaciones, que nos fueron expuestas previamente a la actividad, entre las que se incluían el mantenerse en silencio en todo momento, no estando permitido formar grupos de más de cinco personas, ni moverse con relativa velocidad en el recinto. El respeto para la conservación de las obras es nuestra máxima, con lo que no fue un problema llevarlo a la práctica.

Respecto a las herramientas que el alumnado podía introducir en el recinto expositivo, en cumplimiento de la normativa, depositamos en la taquilla: "los bolsos grandes, paquetes, mochilas, maletines, paraguas u otros objetos que por su tamaño o forma supongan algún peligro para las obras de arte" (Museu Belles Arts València, 2016). Con ello se reducía considerablemente la posibilidad de acceder con carpetas y libretas, con lo que los alumnos entraron a las salas de exposición con sus dispositivos tecnológicos, como tabletas y móviles para la consecución de la didáctica. La normativa indicaba "Está permitido realizar fotografías sin flash y sin trípode, monópode o cualquier elemento de estabilización de cámaras fotográficas" (Ídem, 2016), por lo que el uso de la tecnología se adaptó a estas normas, no resultando un problema debido a la capacidad de reflexión del oro, que impide fotografiarlo con una fuente lumínica directa.

\subsubsection{Sesión de reconocimiento in situ.}

El profesorado hizo una sesión de reconocimiento, para realizar un análisis sistemático de cada una de las obras que podían ser útiles para la actividad, analizando la técnica y manufactura de dorado de las tablas y marcos seleccionados, y estableciendo un listado de deterioros que podían encontrarse sobre los mismos, que estaban alterando la visión actual y la legibilidad de las mismas.

\subsubsection{Preparación de plantillas y fichas STEAM.}

Una vez analizados y seleccionados los casos concretos de análisis, se distribuyó la práctica en dos subapartados, uno relacionado con la descripción y estudio técnico y el otro, con la observación y búsqueda de técnicas en marcos dorados. Como el número de alumnos y alumnas de la asignatura es de 43 , se establecieron 8 modelos de actividades diferentes. 

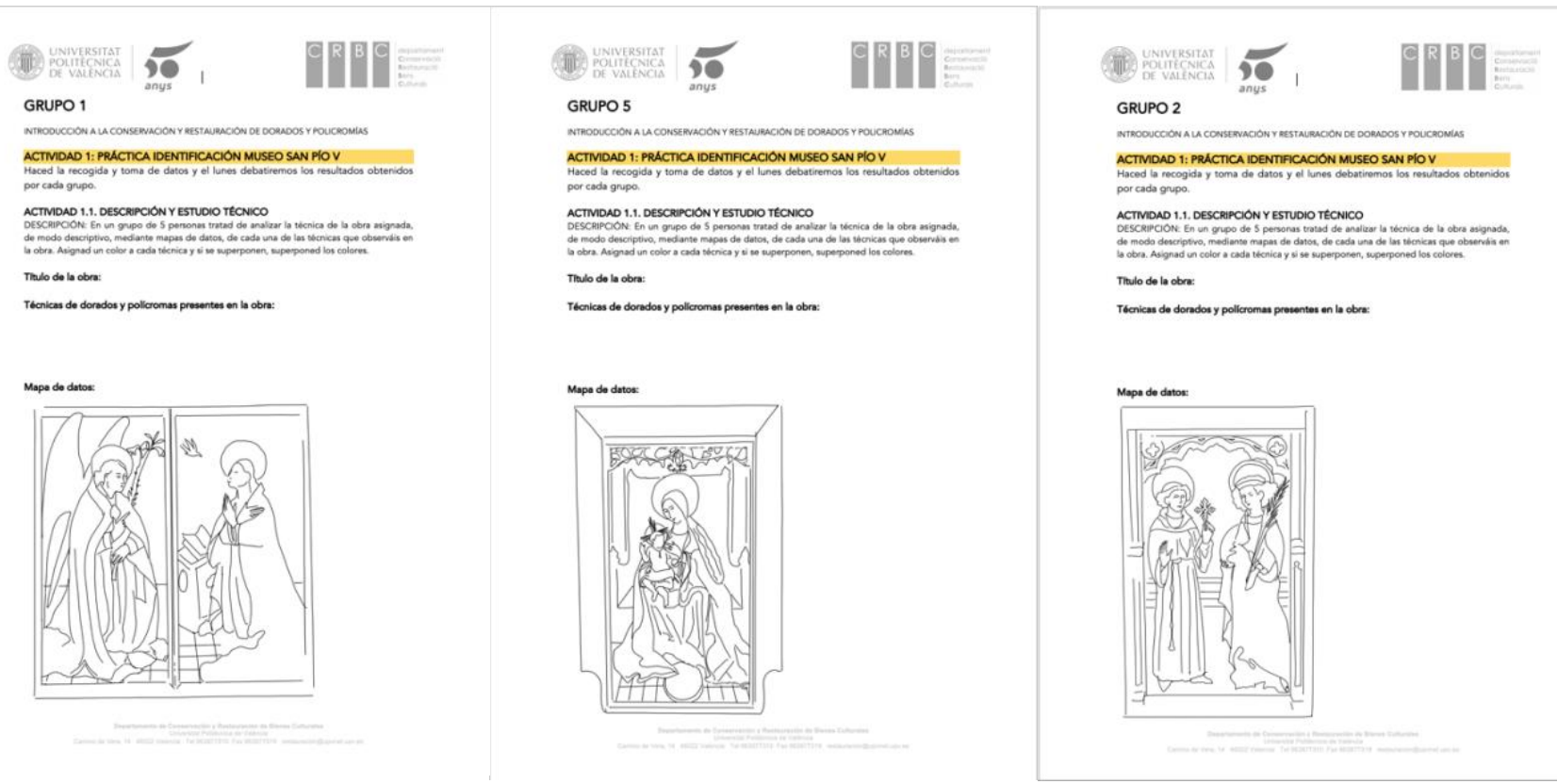

Imágenes 1-3. Ejemplos de modelo de fichas STEAM preparadas por el profesorado. Fuente: Propia.

Las instrucciones eran las siguientes (Carabal, 2020):

“ACTIVIDAD 1.1. DESCRIPCIÓN Y ESTUDIO TÉCNICO. En grupos de hasta 5 personas, tratad de analizar la técnica de la obra asignada, de modo descriptivo, mediante mapas de datos, de cada una de las técnicas que observáis en la obra. Asignad un color a cada técnica y si se superponen, superponed los colores" (idem, 2020).

El profesorado distribuyó una ficha diferente para cada grupo (IMÁGENES 1-3), en el que tenían que buscar la obra que les había tocado analizar, teniendo que marcar en su superficie lo planteado, a través de sus herramientas tecnológicas.

La siguiente parte de la actividad encerraba en sí misma una temática muy compleja para los restauradores, envolviéndola en un planteamiento lúdico. En este caso tenían que recurrir a la observación y la toma de datos individuales, para comprender los procesos por los que han pasado alguna de las obras con el paso del tiempo y que explican su estado de conservación y su estética actual. Con ello se empleó la gamificación y tuvo una percepción por parte del alumnado a modo de escape room, término empleado por ellos mismos para definir la actividad, no perdiendo en ningún momento de vista la normativa del museo y respetando la misma en todo momento.

ACTIVIDAD 1.2. OBSERVACIÓN Y BÚSQUEDA DE TÉCNICAS EN MARCOS DORADOS. En el mismo grupo de 5 personas tratad de buscar las técnicas que se os señalan, tanto en el primer piso como en las diferentes salas del segundo. Apuntad el título de la obra y haced foto al marco que contiene la técnica que tenéis que encontrar." (Ibídem, 2020)

Se les instaba a buscar técnicas específicas como, entre otras:

- MARCO CON ORO FINO BRUNIIDO REDORADO CON ORO MATE (Buscad 1). Explicad en qué os basáis para discriminar esta técnica.

(cc) EY-NC-ND 2020, Universitat Politècnica de València

Congreso In-Red (2020) 
- MARCO ESTOFADO. Explicad en qué os basáis para discriminar esta técnica.

- MARCO CON PINTURA GRASA SUPERPUESTA. Explicad en qué os basáis para discriminar esta técnica.

De este modo, fueron analizando las piezas de las diversas salas, en silencio y tranquilamente, hasta encontrar cada una de las obras que se habían propuesto para cada grupo. En total había 16 preguntas que hacían referencia a cada tabla o marco, específicamente estudiada por el profesorado.

\subsection{Desarrollo de la experiencia docente}

El desarrollo de práctica siguió correctamente el cauce planteado con anterioridad (FIGURA 2). El diseño de la misma seguía los siguientes pasos: la formación de equipos de modo aleatorio, la distribución del trabajo por equipos, el desarrollo presencial de la actividad, el trabajo no presencial de ahondar en la investigación, además de la exposición y debate. Una vez llegados al museo, siguiendo la normativa del mismo, nos facilitaron un espacio donde depositar las mochilas, etc., del alumnado. Únicamente disponían de móvil o cámara para poder realizar la actividad con las fichas en las que tenían que efectuar la misma, preparadas previamente y subidas a la herramienta de la UPV PoliformaT, en varios formatos para facilitar el trabajo. Los formatos eran .doc, .pdf y .jpg.

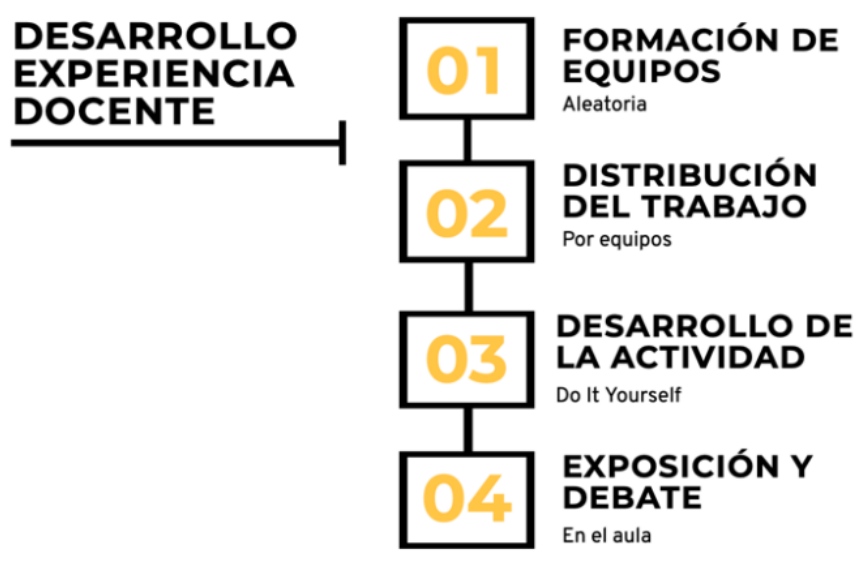

Figura 2. Esquema de la metodología llevada a cabo. Fase 2: desarrollo. Fuente: elaboración propia.

\subsubsection{Formación de equipos}

En la presente actividad, la formación de equipos de trabajo fue en base al orden de llegada del alumnado. Durante las clases presenciales hay grupos formados, en los que se desarrolla el trabajo compartiendo herramientas y materiales con un determinado equipo. Lo que se trataba de conseguir, con una selección aleatoria, es que estos grupos cambiasen en esta ocasión, para poder intercambiar roles y responder a un modo de trabajo no mecánico. No obstante, esta distribución no se realizó de manera rígida, sino flexible, analizando casos específicos en caso de necesidad. 


\subsubsection{Distribución del trabajo por equipos}

Se asignó la numeración de los grupos, con respecto a las fichas STEAM preparadas y puestas a disposicón del alumnado a través de la citada herramienta institucional.

\subsubsection{Desarrollo de la actividad}

Cada equipo diferente empleó la herramienta DIY, para distribuir el tiempo y el espacio, mediante una organización interna en cada grupo e intergrupal, teniendo en cuenta el número máximo de personas en cada uno de los espacios.

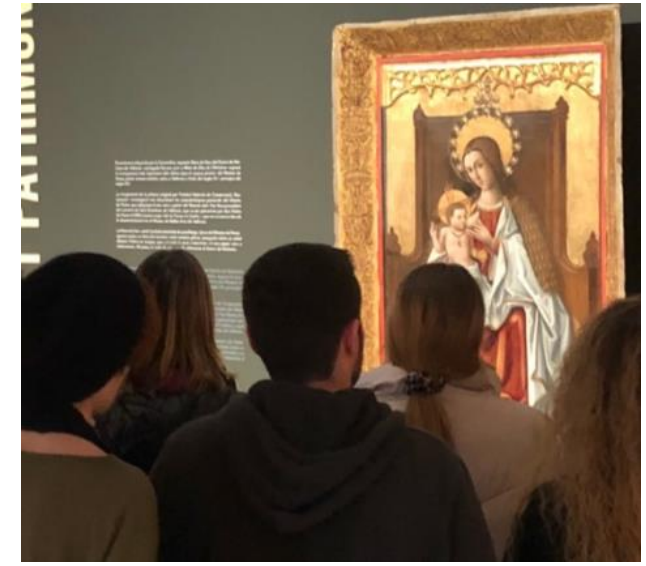

Imagen 4. Desarrollo de la actividad. Fuente: Propia.

\subsubsection{Exposición y debate}

Una vez finalizada la actividad presencial, la experiencia docente precisaba de una puesta en común de los datos tomados por cada uno de ellos, en el entorno del museo. Con ello, se trataba de fomentar el aprendizaje por pares y el trabajo cooperativo. Todo ello para mejorar la toma de datos instantánea, con el objetivo de efectuar la exposición y debate, atendiendo a posibles dudas y efectuando una búsqueda de datos más complejos, mediante la investigación en profundidad de cada una de las obras asignadas a cada grupo.

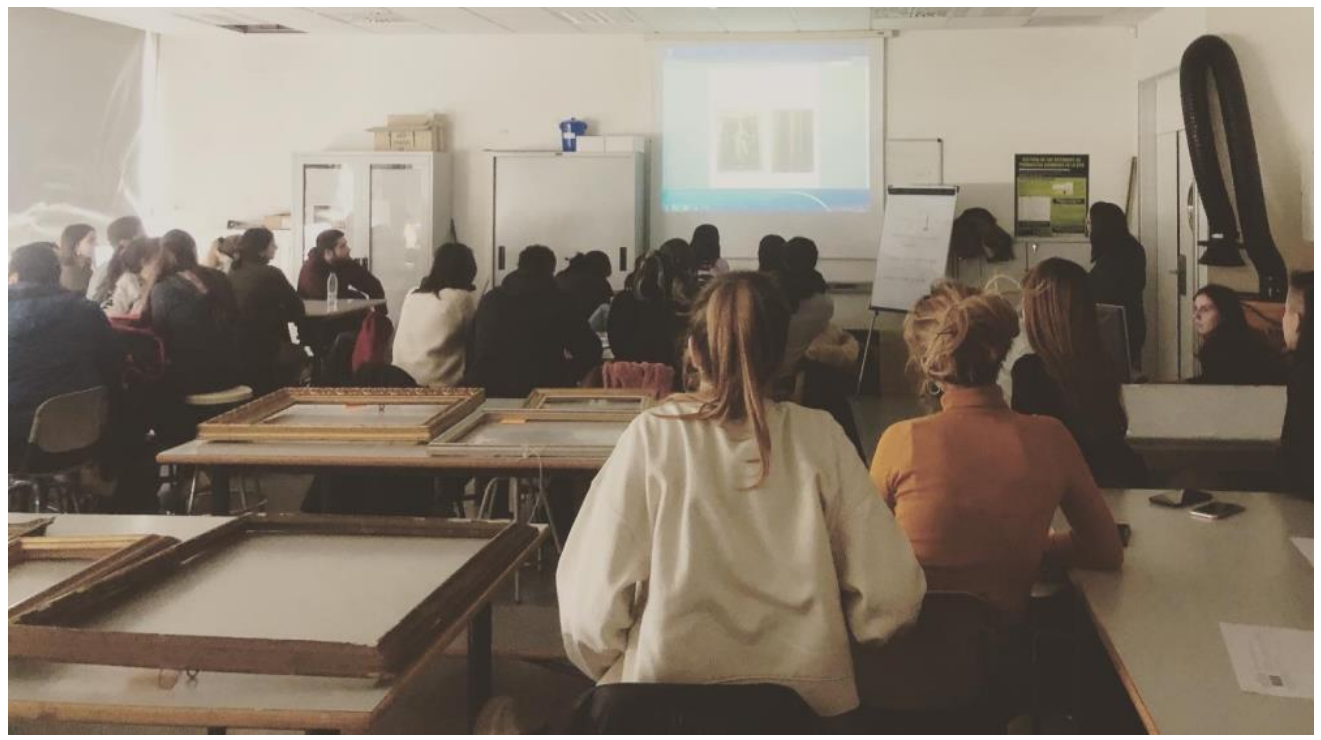

Imagen 5. Exposición y debate en el aula. Fuente: Propia. 


\section{Resultados}

Los resultados obtenidos en la fase de síntesis (FIGURA 3), han comprendido el análisis de resultados obtenidos, la evaluación por parte del alumnado y las propuestas de mejora. El profesorado ha realizado la calificación de la práctica, teniendo en cuenta la adquisición de las competencias y competencias transversales, que se han trabajado en la práctica, que son fundamentalmente: Comprensión e integración, Innovación, creatividad y emprendimiento, Diseño y proyecto, Comunicación efectiva y Conocimiento de problemas contemporáneos.

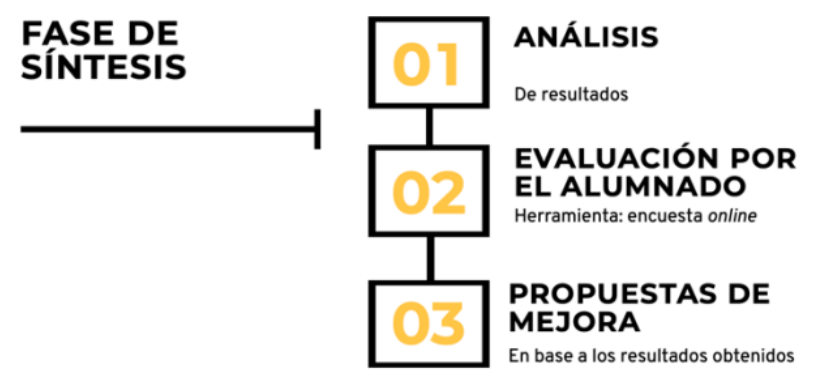

Figura 3. Esquema de la metodología llevada a cabo. Fase 3: síntesis. Fuente: elaboración propia.

\subsection{Fase de síntesis}

\subsubsection{Análisis de resultados}

El primer elemento para poder comprobar y medir el alcance de la práctica, ha sido el resultado y la propia evaluación, mediante análisis objetivos, ahondando principalmente en la adquisición e integración de las competencias y contenidos. Solamente había una respuesta correcta por equipo para la primera pregunta y opciones contadas para la segunda de ellas, con lo que resultó relativamente asequible este primer análisis de adquisición de contenidos.

En segundo lugar, se analizó el nivel de adquisición de las competencias transversales, mediante la elaboración de una rúbrica para cada una de ellas, que iba siendo evaluada por el profesorado en cada fase de la experiencia docente. La elaboración de las rúbricas siguió el patrón establecido por el Instituto de Ciencias de la Educación de la UPV, en su texto Papel de las rúbricas en la evaluación de las competencias transversales UPV (ICE, 2020) en el que se valoraron el nivel del grado de dominio, los indicadores y los descriptores. Respecto a la adquisición de las competencias, fueron evaluadas atendiendo a los resultados de las pruebas objetivas, contenidas en la exposición y el debate, además de un informe con la resolución de los casos planteados, individual y grupal.

\subsubsection{Evaluación por el alumnado}

En segundo lugar se ha recogido una recopilación de resultados del nivel de satisfacción del alumnado, con la herramienta de encuestas online SurveyMonkey® (SurveyMonkey, 1999-2020). Esta encuesta ha sido respondida por el $98 \%$ de los alumnos que efectuaron la práctica, un total de 41 respuestas, que se van a distribuir en porcentajes para el análisis de resultados.

En términos generales la satisfacción global es abrumadora, dado que el 100\% del alumnado considera la práctica como integradora de teoría y práctica, en un escenario fuera de lo cotidiano. 
Desarrollo de la competencia técnológica en el marco STEAM para la docencia universitaria: experiencia en el Museu de Belles Arts de València

\section{Considero que la práctica de identificación en el Museo me sirvió para integrar teoría con práctica de un modo innovador}

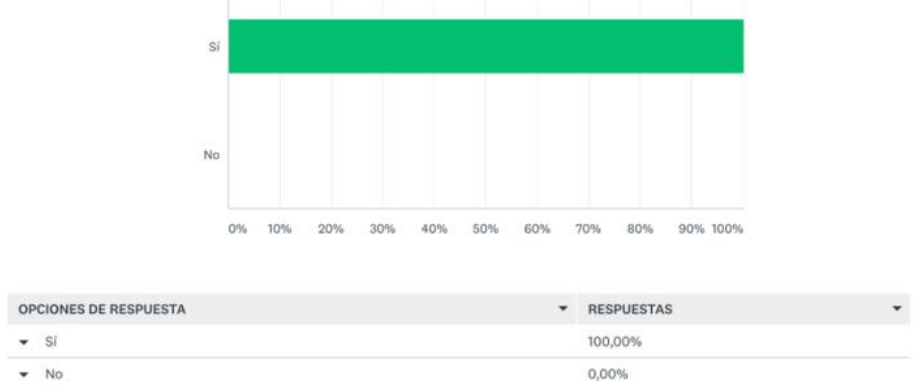

Figura 4. Encuesta con SurveyMonkey®. Fuente: elaboración propia.

Asimismo el $96,30 \%$ del alumnado considera que la práctica fomentaba el trabajo en equipo, el debate y el aprendizaje por pares.

Esta práctica sirvió para fomentar el trabajo en equipo, el debate y el aprendizaje por pares (aprender de mis propios compañeros)
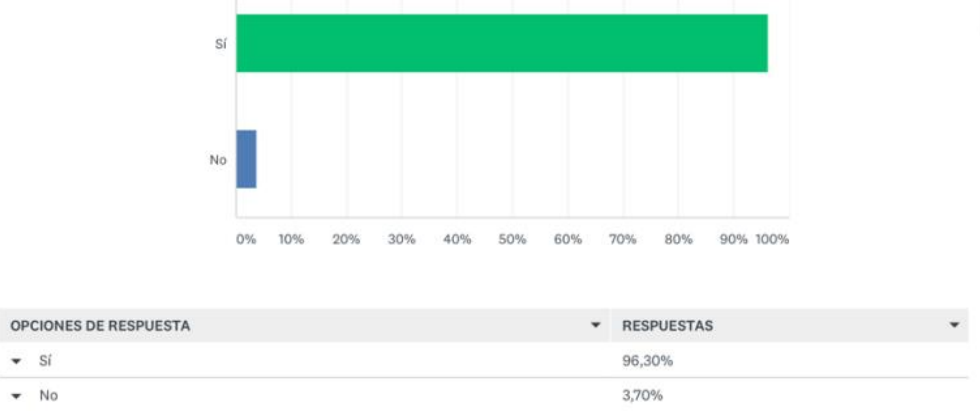

Figura 5. Encuesta con SurveyMonkey®. Fuente: elaboración propia.

Asimismo, el 96,30\% de los encuestados valora de manera positiva la integración del pensamiento flexible y la capacidad de análisis, como herramientas trabajadas.

Esta práctica desarrolla el pensamiento flexible y la capacidad de análisis

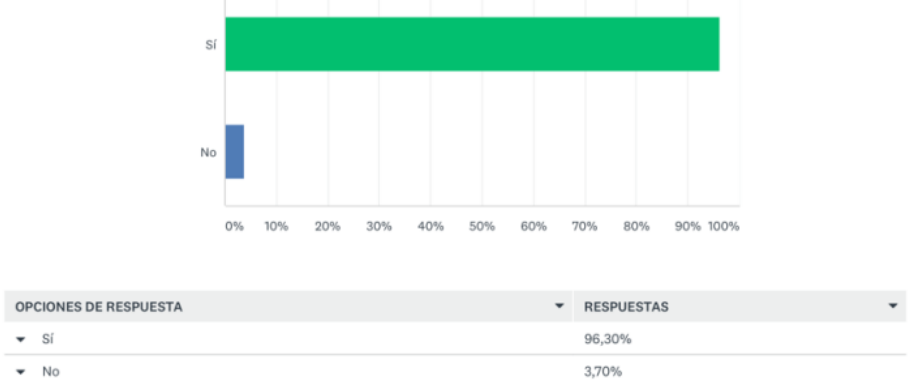

Figura 6. Encuesta con SurveyMonkey®. Fuente: elaboración propia.

El 92, 59\% del alumnado considera la práctica como un ejercicio de gamificación, de hecho el término empleado -escape room- fue empleado por los propios alumnos para definir la experiencia, por supuesto 
salvando las distancias con dichas actividades y con un total respeto por la institución en la que efectuamos la actividad.
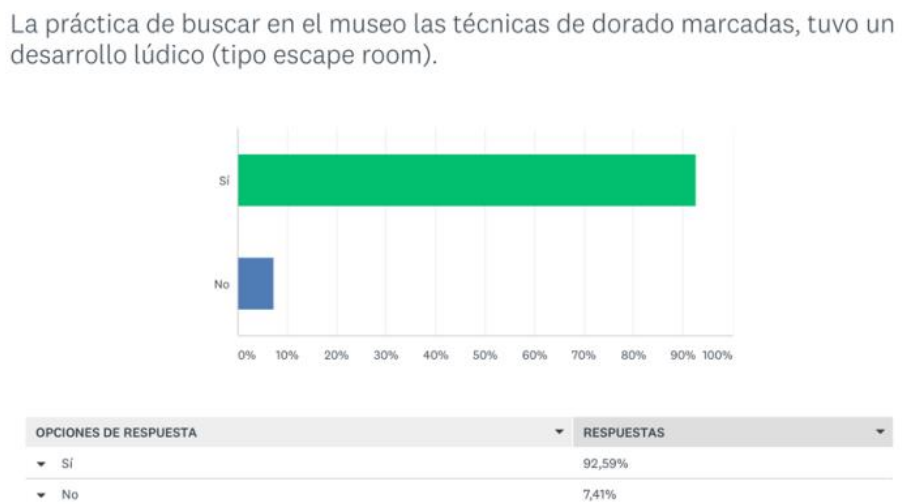

Figura 7. Encuesta con SurveyMonkey ${ }^{\circledR}$. Fuente: elaboración propia.

Respecto a la metodología BYOD, se les preguntó acerca de la posible incomodidad de llevar las herramientas personales para su desarrollo. Un $96,30 \%$ respondió que no resultó un problema.
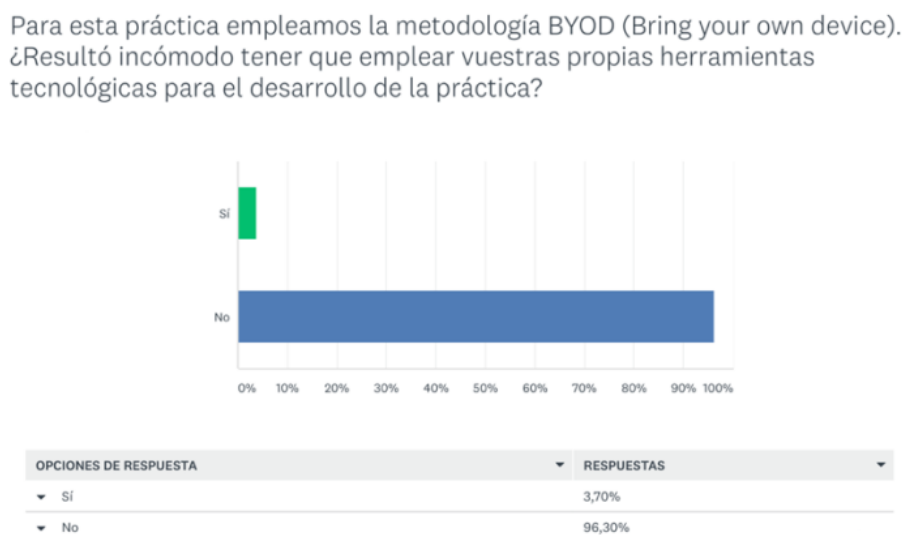

Figura 8. Encuesta con SurveyMonkey®. Fuente: elaboración propia.

Respecto a la herramienta DIY, acerca de la libertad de espacio y distribución del tiempo, un 92,59\% del alumnado la considera adecuada, un 3,70\% considera que no fue adecuada y un 3,70\% indica que hubiera preferido tener más tiempo para la actividad, en el apartado "otros", para no tener que desarrollarla con una percepción de premura. 


\section{¿Te pareció adecuada la libertad respecto a tiempos y a espacios (DIY: Do it Yourself)?}

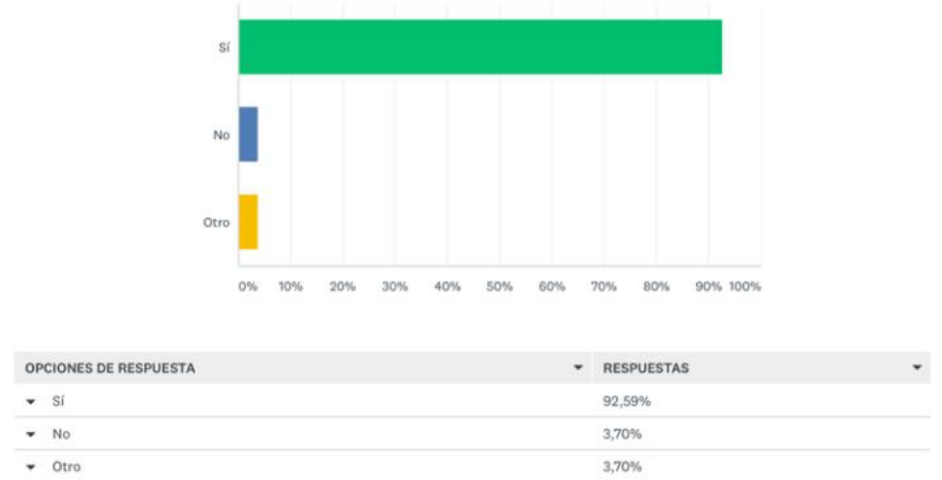

Figura 9. Encuesta con SurveyMonkey®. Fuente: elaboración propia.

Respecto al análisis de tiempos, de gran importancia para el profesorado, a la hora de evaluar de manera significativa la experiencia. El 66,67\% del alumnado reconoce haber empleado unos 30 minutos, un 22,22\% declara haber empleado una hora, mientras que el 11,11\% del apartado "otros" se sitúan en una horquilla intermedia de los 30-60 minutos. La valoración de los tiempos siempre resulta complicado -y más teniendo en cuenta que es una primera experiencia- porque tratamos de integrar a la totalidad del alumnado en una misma franja, pero la diversidad real que tenemos en las aulas, la diferencia de habilidades o destrezas en cada alumno o alumna, nos señala que, en ocasiones, pese a nuestros esfuerzos, el tiempo real no siempre es el que podamos estimar oportuno para cada actividad. Por ello, los docentes universitarios tenemos que trabajar en ese sentido, siendo "necesario adecuar todos los elementos y circunstancias dentro del contexto educacional y del aula para posibilitar el éxito educativo" (Paz Maldonado, 2018).

\section{En la toma de datos empleasteis los siguientes tiempos:}

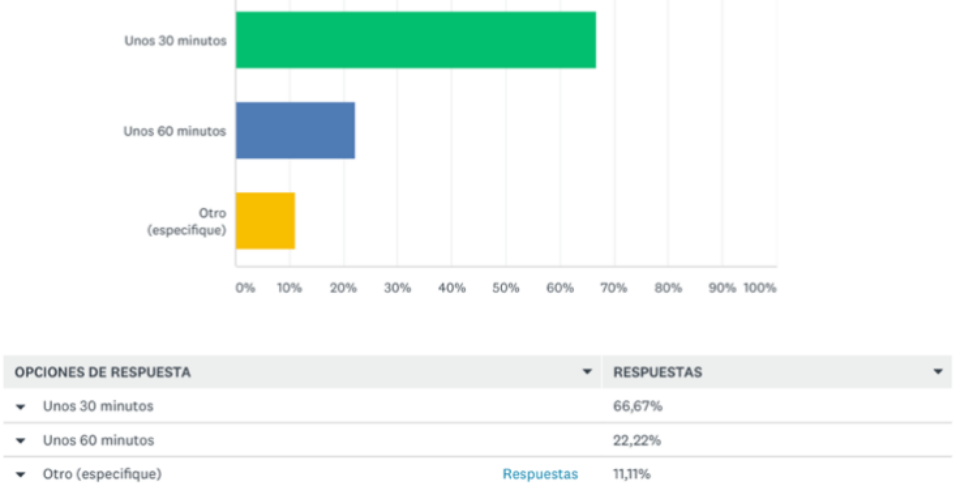

Figura 10. Encuesta con SurveyMonkey®. Fuente: elaboración propia.

Respecto a los tiempos del trabajo no presencial, la media empleada ha sido de unos 30 minutos por alumno/a en un $81,48 \%$ de los casos, mientras que un $18,52 \%$ declara haber invertido 60 minutos en la parte de investigación y trabajo grupal. 


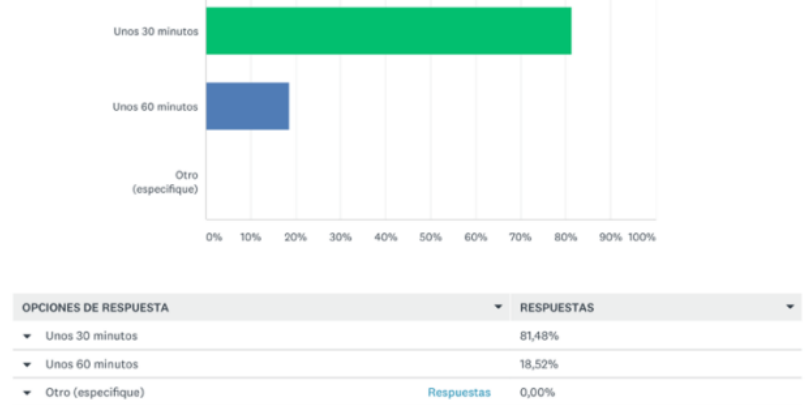

Figura 11. Encuesta con SurveyMonkey®. Fuente: elaboración propia.

Respecto a la valoración de la práctica, el $100 \%$ del alumnado encuestado considera que las herramientas de debate y exposición son útiles para su formación.

Creo las herramientas de debate y exposición son útiles para mi aprendizaje

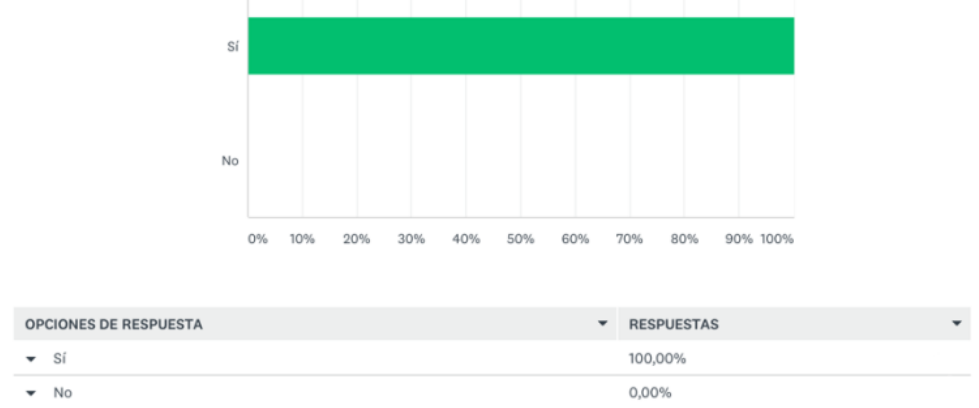

Figura 12. Encuesta con SurveyMonkey®. Fuente: elaboración propia.

Asimismo, el 100\% de los/as alumnos/as considera que deberíamos implantar más actividades de este tipo en la docencia universitaria.

Deberíamos implantar más actividades de este tipo
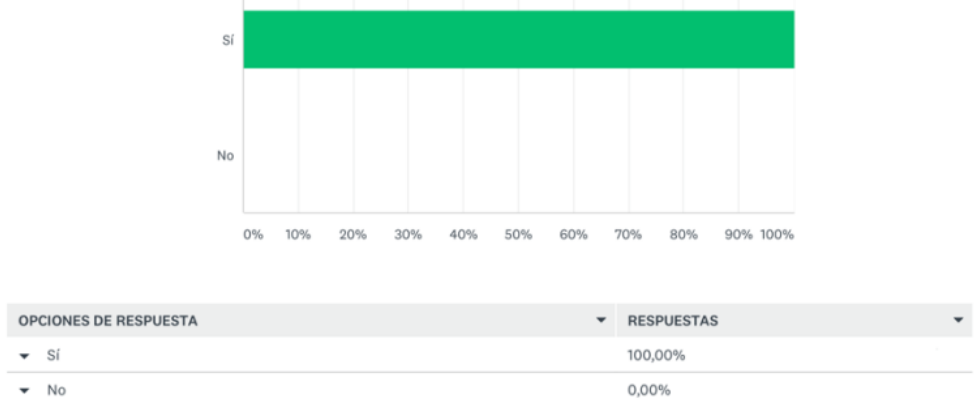

Figura 13. Encuesta con SurveyMonkey®. Fuente: elaboración propia.

(cc) EY-NC-ND 2020, Universitat Politècnica de València

Congreso In-Red (2020) 


\subsubsection{Propuestas de mejora}

Fundamentalmente los resultados y las conclusiones de esta primera páctica, se tendrán en cuenta para cursos académicos posteriores. Las conclusiones extraídas se extrapolarán a la docencia en el futuro, dado que la experiencia docente ha servido de banco de pruebas, analizando diferentes posibilidades para abordar las mismas temáticas, siempre con la aplicación de STEAM, para la docencia del presente y futuro.

\section{Conclusiones}

En el presente trabajo se han analizado los resultados de la eficacia de la aplicación de estrategias STEAM, fuera del aula, gracias al uso de la tecnología. Esta experiencia ha sido valorada como positiva por el $100 \%$ del alumnado, que además, con el mismo porcentaje considera necesaria su implementación en más asignaturas.

La aplicación de herramientas STEAM, en sus diversas competencias, ha supuesto en el alumnado un aumento motivacional, que ha valorado de una manera positiva y ha provocado un avance en los contenidos trabajados. Asimismo, la morfología de la práctica, con una base gamificada, ha sido calificada como un elemento facilitador del aprendizaje, dado que cualquier elemento tecnológico fuera del aula, se adapta a sus códigos visuales y de lenguaje.

La presente práctica ha contribuido a generar en el alumnado una visión analítica y real de los contenidos trabajados, desarrollando el pensamiento práctico y crítico, promoviendo la adquisición de competencias transversales y aplicando los ODS de Naciones Unidas. Asimismo se han desarrollado otros elementos como la promoción del trabajo en equipo, el desarrollo de metodologías cooperativas, liderazgo y la resolución de problemas, potenciando la igualdad de condiciones, la paridad y la diversidad.

La metodología docente que se presenta, no obstante, seguro es transferible a cualquier disciplina universitaria en otros centros o instituciones y con prácticas específicas diseñadas para ello, por este motivo se ha hecho especial hincapié en la difusión de los resultados de la experiencia en medios especializados, para el profesorado interesado en implementar experiencias similares con posterioridad.

\section{Agradecimientos}

Nos gustaría agradecer al Museu de Belles Arts de València todas las facilidades puestas a nuestro alcance para el correcto desarrollo de la práctica.

Este trabajo se ha desarrollado dentro del proyecto de innovación y mejora educativa (PIME) 'Aplicando estrategias STEAM en las áreas de Sociales y Artes, a través de actividades de Aprendizaje-Servicio', coordinado por la profesora María de Miguel Molina, con el apoyo de la Universitat Politécnica de València (Instituto de Ciencias de la Educación, ICE).

Asimismo, las profesoras M.A. Carabal, Virginia Santamarina Campos y Blanca de Miguel pertenecen al Equipo de Innovación y Calidad Educativa (EICE) 'Baños de Realidad: hacia un aprendizaje visible, responsable e innovador', coordinado por la profesora V. Santamarina, y la profesora Marival Segarra pertenece al EICE 'Grupo de Aprendizaje Experiencial'.

\section{Referencias}

AFREEN, R. « Bring Your Own Device (BYOD ) in Higher Education: Opportunities and Challenges» in International Journakl of Emerging Trends \& Technology in Computer Science (IJETTCS). Volume 3, Issue 1, 2014. 
https://www.researchgate.net/profile/Rahat_Siddiqui/publication/261136229_Bring_Your_Own_Device_BYOD_in Higher_Education_Opportunities_and_Challenges/links/54e2dc520cf296663797c13d.pdf [Consulta : 11/03/2020].

ARS MAGNA. 2020. Museo de Bellas Artes San Pío V. < https://arsmagna.es/portfolio-items/museo-de-bellas-artessan-pio-v/> [Consulta: 4 de febrero de 2020] [institucional].

CARABAL MONTAGUD, M.A. 2020. Ficha para la elaboración de la práctica Identificación Museo San Pío V. Material propio de la asignatura Introducción a la Conservación y Restauración de Dorados y Policromías. Grado en Conservación y Restauración de Bienes Culturales. Universitat Politècnica de València.

GARCIA, F. H., ALONSO-CANO, C. 2018. La perspectiva Do It Yourself (DIY) en la enseñanza universitaria. Dar cuenta de las competencias que se aprenden mediante Objetos Visuales Digitales. https://www.researchgate.net/profile/Cristina_Alonso5/publication/329505849_La_perspectiva_Do_It_Yourself_DIY en_la_ensenanza_universitaria_Dar_cuenta_de_las_competencias_que_se_aprenden_mediante_Objetos_Visuales Digitales/links/5c0bb32aa6fdcc494fe32800/La-perspectiva-Do-It-Yourself-DIY-en-la-ensenanza-universitaria-Darcuenta-de-las-competencias-que-se-aprenden-mediante-Objetos-Visuales-Digitales.pdf [Consulta : 25/07/2020]

HURST, Amy; TOBIAS, Jasmine. Empowering individuals with do-it-yourself assistive technology. En The proceedings of the 13th international ACM SIGACCESS conference on Computers and accessibility. 2011. p. 11-18.

INSTITUTO DE CIENCIAS DE LA EDUCACIÓN. UPV. Papel de las rúbricas en la evaluación de las competencias transversales UPV. http:/www.upv.es/entidades/ICE/info/U0702453.pdf [Consulta : 25/07/2020] [institucional].

MUSEU BELLES ARTS VALÈNCIA. 2016. Información práctica. $<$ http://www.museobellasartesvalencia.gva.es/es/informacion-practica $>$ [Consulta : 16/03/2020] [institucional].

OUWENEEL, E., LE BLANC, P. M., SCHAUFELI, W. B. 2013. Do-it-yourself. Career Development International. https://www.researchgate.net/profile/PM_Blanc/publication/270799292_Do-ityourself/links/54b8dae20cf28faced6254c6.pdf [Consulta : 25/07/2020].

PAZ MALDONADO, Eddy Javier. La formación del profesorado universitario para la atención a la diversidad en la educación superior. IE Revista de investigación educativa de la REDIECH, 2018, vol. 9, no 16, p. 67-82.

UNITED NATIONS, Sustainable Development Goals, 2019. https://www.un.org/sustainabledevelopment/ [Consulta : 14/03/2020] [institucional].

UNIVERSITAT POLITÈCNICA DE VALÈNCIA, 2019. Introducción a la Conservación y Restauración de Dorados $y$

Policromías

http://www.upv.es/pls/oalu/sic gdoc.get_content?P_OCW=\&P_ASI=14068\&P_IDIOMA=c\&P VISTA=normal\&P $T I T=\& P \_C A C A=2019 \& P \_C O N T E N T=$ distribucion $>$ [Consulta : 20/03/2020] [institucional].

ROWE AA, BONHAM AJ, WHITE RJ, ZIMMER MP, YADGAR RJ, HOBZA TM, et al. 2011. CheapStat: An OpenSource, "Do-It-Yourself" Potentiostat for Analytical and Educational Applications. PLoS ONE 6(9): e23783. https://doi.org/10.1371/journal.pone.0023783

SANCHEZ, A. V., \& RUIZ, M. P. 2011. Evaluación de competencias genéricas: principios, oportunidades y limitaciones. Bordón. Revista de pedagogía, 63(1), 147-170.

SURVEY MONKEY, 1999-2020. https://es.surveymonkey.com [Consulta : 20/02/2020]

(c)) EY-NC-ND 2020, Universitat Politècnica de València

Congreso In-Red (2020) 